\title{
Dynamic Assessment: Mechanisms Underlying Cognitive Modifiability
}

\section{Hiwa Weisi ${ }^{\star}$ and Khosro Bahramlou}

Department of Literature and Humanities, Razi University, Kermanshah, Iran

"Corresponding author: Hiwa Weisi, PhD in TEFL, Assistant Professor, Department of Literature and Humanities, Razi University, Kermanshah, Iran, Tel: +988334277603; E-mail: hiwaweisi@gmail.com

Received date: Jun 17, 2016; Accepted date: Mar 29, 2017; Published date: April 03, 2017

Copyright: (C) 2017 Weisi $\mathrm{H}$, et al. This is an open-access article distributed under the terms of the Creative Commons Attribution License, which permits unrestricted use, distribution, and reproduction in any medium, provided the original author and source are credited.

\begin{abstract}
Dynamic Assessment, as an umbrella term, covers a myriad of educational approaches that employ instructional interventions in the assessment process to assess a learner's current capabilities, to provide a prognosis of his development potential, to promote the realization of that potential, and ultimately to effect enduring structural changes in the learner's cognition such that the learner would eventually be capable of independent functioning, problem solving, and adaptation to the life circumstances to which he might be exposed. In this position paper, we adopt a three-tier conception of cognitive modifiability at psychological, systems, and cellular levels. At the psychological level, research has clearly demonstrated that cognitive modifiability is a fact of life and that cognition, and by implication, humans are malleable, open systems capable of change for the better or worse. Literature suggests that memory mechanisms of consolidation and reconsolidation at cellular and systems levels could be invoked to explain the cognitive modifiability at psychological level. Neuro-scientific evidence indicates that each time a consolidated memory is recalled and the neuronal ensemble holding that memory is exited, the relevant neurons enter a labile volatile state. Through protein synthesis, release of chemicals, and changes in Neuro-electric signals, these neurons could re-stabilize themselves and reconsolidate a reconstructed and thus, modified version of the original memory. In systems consolidation, molecularly consolidated memories of one brain system transfer to another brain system and undergo a second more time-consuming phase of consolidation there over a period of weeks to years. Reactivation of a memory in the target system temporarily turns it into an unstable trace and sends it to the source system, later to be reconsolidated to a target system memory again.
\end{abstract}

Keywords: Psychological theories; Neurons; Organisms; Neurological bases

\section{Introduction}

In the dynamic approach to assessment, instruction and assessment are fused into a single unity whereby initial performance provides preliminary assessment data and mediational assistance on problem areas constitutes instructional intervention. Assisted performance on problem areas, in turn, helps further fine tune the initial hypotheses about the learner's capabilities and reveal his potential for development. What the learner is able to do with assistance at present reveals something of substance about what he will be able to do on his own in the future. In addition to providing evidenced flashforths of future independent functioning, Dynamic Assessment (DA) diligently works toward the actualization of that future. In other words, the ultimate goal of DA is to help the individual to transcend the confines of his learner/acquirer status and evolve into a thinker/problem-solver capable of adapting to the life circumstances to which he is exposed. This amounts to stating that DA aims to inflict structural changes in the cognitive structure of an individual and turn his inter-mental cognition into an intra-mental one. In the literature, this eventual goal is identified as cognitive modifiability. Cognitive modifiability relates to the possibility of implementing enduring changes in the cognitive structure of an individual for the betterment of his performance and functioning.

Though cognitive modifiability has been attested in numerous research and clinical studies, few attempts have been made in the direction of elucidating the mechanisms underlying cognitive modifiability. This study is one such attempt at explaining the mechanisms of cognitive modifiability which adopts a three-layered conception of cognitive modifiability at psychological (behavioral), systems (modular), and cellular (neuronal/molecular) levels. The effectiveness of DA procedures in instilling cognitive modifiability at clients has been documented in many studies and psychological publication venues abound with such studies. This, we think, is the psychological aspect of cognitive modifiability which seems to have been solidly established. However, the systems and cellular aspects of cognitive modifiability have not been studied as much, mainly because of technological and ethical limitations.

Technological advancements of the past fourteen years have spurred a renewed interest in these aspects of cognitive modifiability. The ensuing studies seem to suggest that memory mechanisms of consolidation and reconsolidation might be implicated in cognitive modifiability. Consolidation refers to the initial process of memory formation and stabilization which turns a short term memory into a long term one. When consolidated memories are retrieved, they enter a transient unstable state. Reconsolidation relates to the process in which these unstable memories stabilize again in order to persist. These mechanisms occur both at cellular and systems levels and could be invoked to explain the cognitive modifiability at behavioral level.

At the cellular level, it is emerging that as an established memory becomes reactivated the neuronal assembly corresponding to that memory undergo some complex molecular changes and enter a labile volatile state which makes them prone to stimulation and modification. These neurons employ a variety of tactics including protein synthesis, release of chemicals, and changes in neuro-electric signals to re-stabilize themselves. Molecular reactions that re-stabilize 
the neurons perform a second function of encoding and reconsolidating a reconstructed and therefore modified copy of the original memory. Depending on a host of factors, many of whom remain unknown to us, the neuronal ensemble might retain, modify, or lose the original memory. Over extended periods of time, molecularly consolidated memories in a specific brain system might detach themselves from that particular system, migrate to a target system, and undergo a second round of consolidation there. This process is referred to as systems consolidation. In systems reconsolidation, reactivation of a target system memory temporarily transfers that memory trace to the source system. In order to persist, this transient source system memory needs to migrate back to the target system again and get molecularly reconsolidated there anew.

\section{Literature Review}

\section{Dynamic Assessment and cognitive modifiability}

Vygotsky, in his socio-cultural theory of mind (SCT), placed the origin of human cognitive development in the social. He postulated that the interaction of an individual with the world and other fellow humans leads to the development of inter-mental cognition. Over time and with experience, this inter-mental cognition is internalized into intra-mental cognition [1]. Inter-mental cognition is mediated through interactions with others and with physical and symbolic artifacts. As cognition becomes intra-mental, mediation occurs at the psychological level rather than the social one [2]. Based on the premise that cognition originates in the social and only later becomes psychological, Vygotsky suggested that social interaction should prevail in all phases of education since any practice to the contrary would run counter to the natural course of cognitive development. This led to the introduction of instructional interventions in the form of mediated interactions to the assessment process and blurred the traditional distinction between instruction and assessment. Various instantiations of Vygotsky theorizations have been developed over time which is collectively referred to as Dynamic Assessment.

DA procedures make a distinction between zone of actual development (ZAD) and zone of proximal development (ZPD) ZAD relates to the things that an individual might be able to do on his own and unassisted. By contrast, ZPD refers to the things that are beyond independent control of an individual, yet the individual might be capable of performing them in mediated interaction with more capable others. In consonance with Vygotsky theorizations, DA practitioners believe that ZPD [2] should precede ZAD [3-5]. Therefore, in mediated interaction with the learner, they attempt to construct a learner specific ZPD and, building on that, to work toward a learner ZAD [6]. To do so, assessors observe learner performance and accordingly construct tentative hypotheses about learner capabilities, provide mediational intervention whenever the learner displays signs of difficulty with an area, and based on learner's responsiveness to mediation modify their initial hypotheses about learner abilities. In addition to providing an estimate of the learner's current capabilities, mediation ally assisted performance on problem areas that are beyond the independent control of the learner yield a substantiated prognosis of the learner's potential for development and, in the meantime, promote the realization of that potential. These flashforths provide evidenced foresights of the learner's future independent functioning and simultaneously help actualize this future [7].

The ultimate goal of DA practices, thus, is to help learners become independently functioning free agents who are capable of handling the circumstances to which they are exposed in their life span, what Feuerstein et al. [8] refer to as 'adaptability to environment'. Feuerstein and Falik [9] suggest that 'thought' is the key factor in the adaptability of human beings to the environment and facing new situations. Kozulin [10] aptly remarks that DA's goal is to help 'learners' become 'thinkers'. For independent functioning, the learner needs to internalize his interactionally acquired cognitive skills and become capable of problem solving and critical thinking on his own. This human propensity to journey from context sensitive 'learning' to context independent 'thinking', Kozulin identifies as cognitive modifiability.

In his theory of Structural Cognitive Modifiability (SCM), Feuerstein [11] postulated that human beings are open systems whose cognitive structures can be modified if they are exposed to conditions which introduce change into their cognitive structures. This unique propensity of human beings enables them to adapt to changing demands in life situations. Two types of human-environment interactions could introduce change into the human's cognitive structure: direct exposure learning and mediated learning experience (MLE) the development of differential cognitive functioning and higher mental processes hinges on these two types of interactions the second of which is the focus of DA procedures. In DA, assessor mediates between the actively engaged learner and the learning experience to modify the learner's thinking skills over time and affect enduring changes in his cognitive structure. This mediation persists until the learner becomes capable of independent functioning and overcomes barriers to change which might relate to genetics, severity of condition, and critical developmental periods [11,12]. Feuerstein et al. [8] pointed out that cognitive modifiability goes beyond the remediation of specific behaviors or skills and aims at the implementation of structural changes that influence the direction and course of cognitive development. Structural changes transcend isolated events and relate to the ways in which human organisms interact with sources of information. Once in place, structural changes determine the learner's course of future development. Thus, maturational changes and isolated changes that result from experiencing a specific set of circumstances are different from cognitive modifiability.

\section{The case for exploring the neurological bases of cognitive modifiability}

Genetics, neurobiology of brain, and human-environment interactions could be invoked to collectively explain the development of cognition in human beings [13-15]. However, competing psychological theories of the 20th century differed greatly in the degree of emphasis they placed on each of these sources of cognitive development. Oftentimes they went to extremes and appealed to only one of these sources to the exclusion of others. Early proponents of the socio-cultural theory of mind (SCM), to which cognitive modifiability subscribes, were no exceptions. In their zeal to counter theories of mind that heavily leaned on genetics or neurobiology of brain, they made every attempt to downplay the role of these sources and to highlight that of the human-environment interactions.

Roger Sperry stated that "as I see it, we are in a battle of paradigms in the history of science, with psychology pitted against the more exact basic sciences that still adhere to the old bottom-up causal determinism and a quantum mechanics view of things" [13]. Like the fruitless mentalist-behaviorist confrontation, this supposed clash of paradigms, we believe, stems from two false assumptions. The first assumption relates to the division of phenomena into immaterial and material, and the assignment of each type of phenomenon to a 
paradigm supposedly best suited to handle it. The second one relates to the irrelatability of the two types of phenomena.

In the first assumption which smacks of Cartesian mind-brain dualism, mind and all its subsidiary constructs including cognitive modifiability are treated as immaterial entities which must be assigned to psychological paradigm. "Information philosophy views the mind as the immaterial information in the brain, which is seen as a biological information processor. Mind is software in the brain's hardware.

The "stuff" of mind is pure information. Information is neither matter nor energy, though it needs matter for its embodiment and energy for its communication" [16]. However, it will quickly come to mind that if mental constructs were immaterial, how they could have been studied in behavioral terms. We would prefer to treat mind as a higher order, abstracted, yet material entity just like time in physics. Time, unlike other materials, cannot be directly seen, yet it can be measured and its passage can be felt. When something is embodied in matter, we need to use energy for its communication, and it is measured in terms of some units, that something is material though in an elevated sense. However, immaterial it might appear; information cannot exist outside of given instantiations in material forms; bits cannot escape the material constraints of the physical devices that manipulate, store, and exchange them. Such an analysis reveals a surprising picture of computing as a material process through and through [17]. Regarding the hardware-software analogy, it must be said that computer hardware will be useless unless there is software to run on it. Similarly, software without hardware to run on it will be as much useless. This view of mind-brain relationship accords with the dialectics of Marxist philosophy which lies at the heart of DA and cognitive modifiability. Following Marx's dialectics, mind and brain might be conceived of as two aspects of a single phenomenon neither of which can exist without the other thereby bypassing the need to consider mind as immaterial [7].

When mind and body are viewed today as a dualism, the emphasis is on the mind, that is to say the information, being fundamentally different from the material brain. Since the universe is continuously creating new information, by rearranging existing matter, this is an important and understandable difference. Matter (and energy) is conserved, a constant of the universe. Information is not conserved; it is the source of genuine novelty [16]. The last sentence of the above quote, however, seems to be contentious in our view. If we can create new information by rearranging existing matter, disturbing the ensuing arrangement will not necessarily lead to the irrecoverable erasure of that information. It will, just, create a different configuration of matter which might represent a different piece of information. If the original arrangement is reconstructed, the information will be restored. In response to those who might doubt the possibility of reconstructing the original arrangement of matter purporting to a piece of information, it must be said that deletion of any information requires us to disturb its corresponding arrangement of matter. It follows that disturbing any arrangement of matter will leave traces or signatures on the pieces of matter that comprise the arrangement. These traces and signatures would allow us to reconstruct a close enough replica of the original arrangement and by implication restore a reconstructed version of the original information. There are data recovery software that do this in the case of computers. Had it not been for the traces that are left behind after any disturbance of arrangements of matter, the whole science of forensics would have been pointless and escape literature would have lost one of its most productive genres: the detective stories [17]. Therefore, information may be considered a conserved constant, too. And this may be the case with the recovery of old long forgotten information or the loss of unused information in human beings which has sparked the memory loss or memory retrieval debate in psychology. This debate relates to the loss of information or lack of access to information in cases of forgotten information [3-5]. It may be that memories encoded in the brain are never erased and just the arrangements of the units holding those memories are disturbed leading to forgetting. In some certain circumstances such as stimulation of brain areas during surgery or after suffering stroke long forgotten memories have come to the fore of the patients' consciousness. It is tempting to postulate that deliberate or accidental stimulation has made the brain to conjure up the original arrangement of units representing a memory and to retrieve that long forgotten memory.

In contrast to mind, brain and its neurobiology are treated as material entities which should be assigned to neuroscience as a branch of exact sciences. Brain's systems and cellular structures are certainly material, but this does not rule out the possibility that complex activation and interaction patterns at cellular and systems levels of the brain could lead to the formation of an apparently immaterial mind through increasing sophistication and layered abstraction [17]. Neuroscientific evidence has reported many neurological correlates for various mental functions and abilities [18-26].

These studies indicate that the activation of certain neurological circuitries leads to the formation of specific mental functions. Further evidence for the role of brain circuitry in the formation of mental functions comes from the cases in which strokes have caused brain damages and have led to psychological, emotional and behavioral changes that affected the sufferers' personalities [27-30].

Relinquishing the irrelatability assumption allows us to bring the apparently immaterial mind into contact with material brain, and to explain insights gained from psychological paradigm in terms of findings that have emerged from exact sciences paradigm. Therefore, we believe that paradigm wars should be avoided at all costs since these paradigms represent different ways of looking at and explaining the worldly phenomena which must be brought together to afford us a more powerful and comprehensive understanding of the world. Any attempt at proving the worth of one approach to the detriment of the other would leave us an inevitably incomplete picture of the object of study.

The difference between these two paradigms is one of scale, with psychology representing the macroscopic view of phenomena and exact sciences standing for the microscopic view of the same phenomena. Any one in his right mind would agree that psychological reality of mind depends on the specific neurobiology of human brain and its subsystems, and that brain can only be studied by adopting a bottom up quantum mechanics view [14]. Psychology, in its broadest sense, represents a macro scale view of cognition, and by implication cognitive modifiability, at behavioral level. However, it is just that, a macro scale view of cognition which does not obviate the need for looking at cognitive modifiability from other vantage points. A bottom-up micro scale view of cognitive modifiability might be as enlightening as the macro scale view. Had it not been for the bottomup causal determinism and a quantum mechanics view of things, we would not have had much of the technologies that we cannot live without today. We would have remained a tool using species which would have employed whatever that worked without knowing why that worked. We would have used boats without knowing what mechanisms kept them afloat. Thus, we need to explore the underlying 
mechanisms of cognitive modifiability which has been established as a working psychological reality. Feuerstein, a prominent authority on cognitive modifiability, in his more recent works has felt the need for exploring the microscopic of cognitive modifiability and has called for investigating the neurobiological bases of cognitive modifiability [12]. Microscopic view of cognitive modifiability requires the study of cellular structures and component systems of the human brain.

In this spirit, we have adopted a three-tier conception of cognitive modifiability at psychological (behavioral), cellular (neuronal/ molecular), and systems (modular) levels. In our view, DA relates to the psychological aspect of cognitive modifiability. Psychological literature is replete with studies that document the success of DA procedures in affecting enduring structural changes in clients' cognitive structures [31-41]. These practices have enabled clients to overcome problems which were related to genetics, severity of condition, and critical developmental periods. The psychological reality of cognitive modifiability might be explained by making recourse to the functioning of the cellular structures and component systems of human brain [12].

\section{Mechanisms Underlying Cognitive Modifiability}

It was argued that mind and brain are two dialectically related aspects of a single phenomenon which might be identified as cognition. A dialectical relationship between mind and brain dictates that changes in either of these two entities will necessarily implicate modifications in the other. This means that changes in brain might lead to modifications in the mind and, in return, modifications in mind might affect changes in the brain. Performance-based DA procedures affect enduring changes in the mental aspect of a client's cognition. Following the above line of argument, modifications in a client's mental capabilities, identified as cognitive modifiability, must be correlated with some changes in the individual's brain. Neuroscientific literature indicates that, indeed, any mental activity leads to some modifications in areas of the brain which are tasked with encoding and handling that specific mental activity. This tendency of the brain to undergo changes is referred to as plasticity. Therefore, plasticity might be regarded as the brain correlate of cognitive modifiability. Plasticity is made possible through memory formation and retrieval mechanisms of consolidation and reconsolidation which take place at the cellular and systems level of brain.

Okano et al. [42] define learning "as a process for acquiring memory" (p. 12403) Wickens [43] and, Crystal and Glanzman [44] define learning as the acquisition of new information, and memory as the capacity for storing and retrieving the information. He goes on to state that learning and memory as intimately interwoven entities are constantly updated and modified throughout the life provided that individuals actively participate in thought processes. From the close relationship between learning and memory, and their modifiability, one can deduce that cognitive modifiability as the end result of learning at the psychological level could be explained in terms of dynamic memory formation and retrieval mechanisms.

Neurons as the cellular units of brain communicate with each other through synapses. When an organism undergoes a new experience, a network of neurons (cell assembly) is tasked with encoding a memory trace of that experience. Recurrent neural activity of the cell assembly creates a short term memory trace (STM) in the synaptic connections. This trace is labile and open to retention or disturbance depending on the circumstances [45]. During learning, synapses change in ways that make it easier for connected neurons to communicate with each other. This plasticity which forms the basis of memory formation and retention involves activity-driven functional and structural remodeling of neural networks activated during learning. Functional remodeling refers to the modifications of synaptic strength through increasing the chemical signals sent or received by connected neurons. In structural remodeling, a synapse changes in size or shape [46]. With time and in the absence of any disturbance, functional remodeling and morphological alterations of the network's synapses render the synaptic connections of the cell assembly permanent. As synaptic connections become permanent, a long-term memory (LTM) of the experience is encoded. Neuro-scientific literature uses the term 'consolidation' to refer to this process. The time lapse between the onset of an STM and the conclusion of an LTM is referred to as consolidation interval. During this interval, introduction of electroconvulsive shock, protein synthesis inhibitors, and new learning tasks leads to the disturbance of memory or the impairment of performance. By contrast, administering certain compounds such as strychnine enhances the retention of the memory. However, after the consolidation of an LTM, none of these treatments would have any effect on the memory in question [45].

The reactivation of consolidated memories makes them enter a transient labile state which turns them susceptible to amnesic and memory enhancement treatments. In this regard, reactivated memories resemble the short term memories which are in the process of turning into long term consolidated memories. As long as these memories are active, they are open to retention, modification, or disruption. In order to persist, these labile reactivated memories must undergo a second, albeit less time-consuming, round of consolidation which is dubbed as re-stabilization or reconsolidation [45]. However, caution that not all memories can undergo reconsolidation since there are constraints on reconsolidation. These constraints, also called boundary conditions, relate to "situations of physiological, environmental, or psychological nature, in which memory that normally would reconsolidate no longer does".

Debiec et al. [47] argue that it is more parsimonious to conceive of consolidation and reconsolidation as two instantiations of a single activity-driven memory formation mechanism. To support their argument, they reason that both instantiations require that memory should be active, for the consolidation during the process of turning STM to LTM and for reconsolidation shortly after reactivation of an established memory. Further, memories which are undergoing consolidation or reconsolidation are prone to amnesic treatments (such as electroconvulsive shock, protein synthesis inhibitors, and competing learning tasks) and memory enhancement treatments (administration of various compounds, such as strychnine) "The function of this form of ongoing plasticity has not been clarified yet but may well serve to update or modify existing memories" [46].

Bruel-Jungerman et al. [46] accord with Debiec et al. [47] and Hardt et al. [45] in considering consolidation and reconsolidation as subsidiary instantiations of an overarching memory formation and retrieval mechanism. They argue that activity-dependent functional and structural remodelings of synaptic connections act as the neurobiological substrate of this overarching memory formation process. Functional remodeling relates to changes in the chemical and molecular configuration of the synaptic connections that increase or weaken the efficacy of synaptic connections. These changes might lead to Long Term Potentiation (LTP) or Long Term Depression (LTD) which correspond to synaptic strengthening and synaptic weakening 
Page 5 of 7

respectively. In the case of a newly formed memory, changes in the chemical and molecular configuration of the synapses modify the efficacy of synaptic connections holding that memory and lead to retention or disturbance of that trace. In the case of an already consolidated memory which has been reactivated, a second, and often shorter, round of chemical and molecular changes modifies the strength of the synaptic connections and thereby encodes a modified copy of the original memory.

Structural remodeling, on the other hand, relates to the changes in size or shape of synapses. Bruel-Jungerman, Davis and Laroche [46] identify synaptogenesis and synaptic remodeling, and neurogenesis as two types of structural remodeling. Under specific conditions, neural activity stimulates the neurons to grow new synapses in their contact points with other neurons, to develop spines and appendages on their dendrites, or to change their shape. In other circumstances, brand new neurons are born as a result of neural activity which might take on several functions in the memory formation process. However, most of these neurons do not reach maturation and, therefore, disintegrate. Structural remodeling can occur in the initial memory consolidation or in the later reconsolidation of a reactivated memory. When synaptic connections pertaining to a reactivated memory undergo morphological modifications, it is taken as a sign that a modified or updated copy of that memory is being reconsolidated.

Memory consolidation at cellular level is made possible through the activation of various protein kinases or protein phosphatases which control and regulate protein synthesis and gene transcription or repression. Molecular structures produced through this protein synthesis and gene transcription/repression help synapses to grow and strengthen or retract and weaken leading to persistent modification of neural circuits constituting a memory. The same processes are implicated in the reconsolidation of a memory. In certain respects, though, there are some differences between consolidation and reconsolidation [44].

There are some areas in the brain which have been pre-wired to hold and process memories pertaining to certain types of experiences or to certain aspects of complex experiences. These areas are referred to as brain systems. Different aspects of a complex experience might be stored and handled in different systems. Therefore, each system needs to work in parallel and communicate with other systems to encode and recall memories of complex experiences. Consolidation and reconsolidation can be observed at the systems level as well. In the systems consolidation, Recall of memories in the hippocampusdependent declarative memory system becomes gradually-although not necessarily completely-independent of hippocampal networks over time to rely mainly on neocortical networks. Recent fMRI studies have provided evidence that the hippocampo-to-neocortical redistribution of memory representations is promoted by sleep. In parallel to these qualitative changes in the representation of a memory at the neuronal level sleep produces qualitative changes in the memory at the behavioral level. In fact, there is convergent evidence for the notion that the system consolidation process during sleep supports the extraction of invariant and repeating features in newly encoded memories, and in this way, the conversion of implicit into an explicit and conscious form of memory [10]. It assumes two separate memory stores, one that learns at a fast rate and holds information only temporarily, and the other that learns at a slow rate but shows also a slow rate of forgetting and serves as the long term store. New information is initially encoded in parallel into both the temporary and the long-term store. In subsequent periods of consolidation, the newly encoded memory traces are repeatedly reactivated and thereby become gradually reorganized such that the representations in the slow-learning long-term store are strengthened. Through the repeated reactivation of new in conjunction with related older memories, the temporary store acts like an internal "trainer" of the slowly learning long-term store to gradually adapt the new memories to the preexisting knowledge networks. The reactivation and redistribution of memories to the long-term store can also promote the extraction of invariant and relevant features from the new memories, whereas irrelevant features may be erased. Because both stores are used also for encoding of information, this encoding could interfere and disturb the proper consolidation process. Therefore, to prevent such interference, the reactivation and redistribution of memories during consolidation take place in offline periods, i.e., during sleep when there are no encoding demands. In the declarative memory system the fastlearning, temporary and slow-learning long-term stores are represented by the hippocampus and neocortex, respectively. Based on the repeated reactivation of temporary memories in the hippocampus, these are gradually redistributed over periods of days and years to neocortical networks and can ultimately lose their dependence on hippocampal networks. Declarative memory is commonly divided into semantic memory that refers to general knowledge and episodic memory that refers to individual events consciously experienced during wakefulness. Semantic memories in the process of consolidation can in fact become entirely independent from the hippocampus and neighboring medial temporal lobe structures implicating that they are erased from hippocampus networks. By contrast, for episodic memories hippocampal function may be continuously required even after years of consolidation.

Crystal and Glanzman [44] the consolidation of some memories in the mammalian brain involves, in addition, a time dependent transfer of information from one brain region, the hippocampus, to another, the medial prefrontal cortex. The functional reason for this information transfer is unclear, as is whether the transfer is permanent, as proposed by some or whether instead, as Preston and Eichenbaum argue, memories can reside permanently in both regions, thereby allowing the two memory representations to interact under some circumstances.

\section{Discussion}

Cognitive modifiability which results from the administration of mediated learning through DA procedures bears striking resemblances to its brain correlate plasticity. In mediated learning, the learner is actively engaged in performance on an assessment task with the help of an assessor. The assessor observes the learner and, whenever the learner shows signs of difficulty with an area, intervenes in the assessment process to offer needed assistance. This mediational help is contingent, graduated, and dialogic. It is offered only when needed. It is graded in terms of amount and explicitness. It takes place between a more capable tutor and a less able learner [1]. As assisted performance on assessment task proceeds and the learner shows signs of progress on the problem area and is able to maintain his fault-free performance in other similar tasks across various DA sessions, it is argued that the learner has acquired or learned something about the problem area. However, this is different from achieving cognitive modifiability. To achieve cognitive modifiability, the learner should transcend the knowledge that he has gleaned from the learning task to other tasks that are removed from the learning context in terms of complexity, modality and operations. For this purpose, during transcendence 
sessions, the learner is required to perform on near, far, and very far transfer tasks which turn the initial context-specific learning into a context-independent knowledge [10].

Meantime that he assesses the learner (activity driven, immediate intervention in trouble spots, DA vs. TR sessions=transfer=systems consolidation, reconsolidation) A brain system's feeding into and being fed by other brain systems reminds us of the modular view of language processing. In modular view of language processing, each aspect of the linguistic input/output is assigned to a module which has been dedicated to processing that aspect of linguistic input/output.

Without either of these two, there will be no cognition. Units of matter which compose the brain can be configured in various ways to create units of information which compose the mind. In reverse, units of information composing the mind can be manipulated to introduce change into the arrangement of units of matter composing the brain. In line with the above argument and in order to arrive at a better understanding of cognitive modifiability, both mental and neurological aspects of this phenomenon must be investigated. However, the DA literature has unilaterally attended to mental aspect and has neglected the neurological aspect.

This analysis does not draw a clear demarcation between consolidation and retrieval processes and in this view; it can be assumed that every retrieval operation should trigger a reconsolidation process. Moreover, decoding or retrieval will change the information content of the "trace" such that memory can be viewed from a neurobiological point of view as an emergent, dynamic, adaptive property of the nervous system.

Crystal and Glanzman [44] the function of reconsolidation appears to be to provide the ability to respond flexibly to an ever-changing environment; reconsolidation permits an organism to update its memories, either strengthening or weakening them, without having to undergo re-exposure to the original learning situation. One prospect for the future is the goal of integrating a deep understanding of biological mechanisms with sophisticated models of human cognition.

\section{Conclusion}

Literature seems to suggest that memory mechanisms of consolidation and reconsolidation which take place at both cellular and systems levels of brain stand a good chance of explaining the cognitive modifiability which results from the application of DA procedures.

\section{References}

1. Aljaafreh A, Lantolf JP (1994) Negative Feedback as Regulation and Second language Learning in the Zone of Proximal Development. The Modern Language Journal 78: 465-483.

2. Lantolf JP, Poehner ME (2008) Dynamic Assessment. In Encyclopedia of language and education, (2nd edn.), Springer Science+Business Media, LLC, New York.

3. Lewis DJ (1979) Psychobiology of active and inactive memory. Psychological Bulletin 86: 1054-1083.

4. Miller RR, Springer AD (1974) Implications of recovery from experimental amnesia. Psychological Review 81: 470-473.

5. Spear NE (1973) Retrieval of memory in animals. Psychological Review 80: 163-194.

6. Kozulin A, Garb E (2001) Dynamic assessment of EFL text comprehension of at-risk students. Paper presented at the 9th Conference of the European Association for Research on Learning and Instruction, Fribourg, Switzerland.

7. Poehner ME (2011) Validity and interaction in the ZPD: Interpreting learner development through L2 Dynamic Assessment. International Journal of Applied Linguistics 21: 244-263.

8. Feuerstein R, Feuerstein RS, Falik LH, Rand Y (2006) Creating and enhancing cognitive modifiability: The Feuerstein Instrumental Enrichment program. Jerusalem: ICELP Publications.

9. Feuerstein R, Falik LH (2010) Learning to think, thinking to learn: A comparative analysis of three approaches to instruction. Journal of Cognitive Education and Psychology 9: 4-20.

10. Kozulin A (2011) Learning potential and cognitive modifiability. Assessment in Education: Principles, Policy \& Practice 18: 169-181.

11. Feuerstein R (1990) The Theory of structural cognitive modifiability. In: McClure R (eds.) Learning and thinking styles, Classroom interaction Washington DC, National Education Association and Research for Better Schools.

12. Feuerstein R, Falik LH (2013) Structural cognitive modifiability and neural plasticity: Confronting the possibilities. In: Feuerstein $\mathrm{R}$ Feuerstein R (eds.), Proceedings of the Jerusalem International Conference on Neural Plasticity and Cognitive Modifiability, Bologna, Italy.

13. Presseisen BZ, Sternberg RJ, Fischer KW, Knight CC, Feuerstein R (1990) Learning and thinking styles: Classroom interaction. In: McClure R (ed.) Washington DC: National Education Association and Research for Better Schools.

14. Ames DL, Fiske ST (2010) Cultural Neuroscience. Asian Journal of Social Psychology 13: 72-82.

15. Chiao JY, Ambady N (2007) Cultural neuroscience: Parsing universality and diversity across levels of analysis. In: Kitayama S, Cohen D (eds.), Handbook of Cultural Psychology. Guilford Press, NY 237-254.

16. Information philosopher (2014) The Mind-Body Problem.

17. Blanchette JF (2011) a material history of bits. Journal of the American Society for Information Science and Technology 62: 1042-1057.

18. Chechko N, Drexler EI, Voss B, Kellermann T, Finkelmeyer A, et al. (2014) Neural correlates of unsuccessful memory performance in MCI. Frontiers in Aging Neuroscience Art 201: 1-11.

19. Gruber O, Indefrey P, Steinmetz H, Kleinschmidt A (2001) Dissociating Neural Correlates of Cognitive Components in Mental Calculation. Cerebral Cortex 11:350-359.

20. Herholz SC, Halpern AR (2012) Neuronal Correlates of Perception, Imagery and Memory for Familiar Tunes. Journal of Cognitive Neuroscience 24: 1382-1397.

21. Miller EK, Nieder A, Freedman DJ, Wallis JD (2003) Neural correlates of categories and concepts. Current Opinion in Neurobiology 13: 198-203.

22. Platek SM, Keenan JP, Gallup GG, Mohamed FB (2004) Where am I? The neurological correlates of self and other. Cognitive Brain Research 19: 114-122.

23. Potvin S, Bourque J, Durand M, Lipp O, Lalonde P, et al. (2013) The Neural Correlates of Mental Rotation Abilities in Cannabis-Abusing Patients with Schizophrenia: An fMRI Study. Schizophrenia Research and Treatment, pp: 1-10.

24. Rabin JS, Gilboa A, Stuss DT, Mar RA, Rosenbaum RS (2010) Common and unique neural correlates of autobiographical memory and theory of mind. Journal of Cognitive Neuroscience 22: 1095-1111.

25. Shelton AL, Gabrieli JDE (2004) Neural Correlates of Individual Differences in Spatial Learning Strategies. Neuropsychology 18: 442-449.

26. Zuk J, Benjamin C, Kenyon A, Gaab N (2014) Behavioral and Neural Correlates of Executive Functioning in Musicians and Non-Musicians. PLoS ONE 9: 1-14.

27. Attewill F (2011) Rugby player suffers stroke and then becomes gay hairdresser.

28. Dixon H (2013) Stroke victim unable to feel sadness. The Telegraph.

29. Khateb A, Simon SR, Dieguez S, Lazeyras F, Blanke O, et al. (2009) Seeing the phantom: A functional magnetic resonance imaging study of a supernumerary phantom limb. Annals of Neurology 65: 698-705. 
Citation: Weisi H, Bahramlou K (2017) Dynamic Assessment: Mechanisms Underlying Cognitive Modifiability. Abnorm Behav Psycho 3: 130. doi: $10.4172 / 2472-0496.1000130$

Page 7 of 7

30. Walters K (2008) My stroke made me an artist. The Guardian.

31. Feuerstein R (1980) Instrumental enrichment. Baltimore, MD: University Park Press.

32. Feuerstein R (1981) Cognitive modifiability in adolescence: Cognitive structure and the effects of intervention. The Journal of Special Education 15: 269-287

33. González HL, Palencia AP, Umaña LA, Galindo L, Villafrade MLA (2008) Mediated Learning experience and concept maps: a pedagogical tool for achieving meaningful learning in medical physiology students. Advances in Physiology Education 32: 312-316.

34. Hadas-Lidor N, Katz N, Tyano S, Weizman A (2001) Effectiveness of Dynamic cognitive intervention in rehabilitation of clients with schizophrenia. Clinical Rehabilitation 15: 349-359.

35. Hickson J, Skuy M (1990) Creativity and Cognitive Modifiability in Gifted Disadvantaged Pupils: A Promising Alliance. School Psychology International 11: 295-301.

36. Keane KJ, Kretschmer RE (1987) Effect of Mediated learning intervention on cognitive task performance with a deaf population. Journal of Educational Psychology 79: 49-53.

37. Kozulin A, Lebeer J, Madella-Noja A, Gonzalez F, Jeffrey I, et al. (2010) Cognitive modifiability of children with developmental disabilities: A multicenter study using Feuerstein's Instrumental Enrichment--Basic program. Research in Developmental Disabilities 31: 551-559.

38. Lifshitz H, Rand Y (1999) Cognitive modifiability in adult and older people with mental retardation. Mental Retardation 37: 125-138.
39. Skuy M, Apter A, Dembo Y, Tyano S, Kaniel S, et al. (1992) Cognitive modifiability of adolescents with schizophrenia: A research note. Journal of Child Psychology and Psychiatry, and Allied Disciplines 33: 583-589.

40. Tzuriel D, Kaufman R (1999) Mediated learning and cognitive modifiability: Dynamic assessment of young Ethiopian immigrants in Israel. Journal of Cross-Cultural Psychology 30: 359-380.

41. Tzuriel D. Weiss S (1998) Cognitive modifiability as a function of mother-child mediated learning strategies, mothers' acceptancerejection, and children's personality. Early Development and Parenting 7: 79-99.

42. Okano H, Hirano T, Balaban E (2000) Learning and memory. Proceedings of the National Academy of Sciences 97: 12403-12404.

43. Wickens AP (2005) Foundations of Biopsychology (2nd edn), Pearson/ Prentice Hall, New York.

44. Crystal JD, Glanzman DL (2013) A biological perspective on memory. Current Biology 23: R728-R731.

45. Hardt O, Einarsson EO, Nader K (2010) A bridge over troubled water: Reconsolidation as a link between cognitive and Neuroscientific memory research traditions. Annual Review of Psychology 61: 141-167.

46. Bruel-Jungerman E, Davis S, Laroche S (2007) Brain plasticity mechanisms and memory: A party of four. Neuroscientist 13: 492-505.

47. Debiec J, LeDoux JE, Nader K (2002) Cellular and systems reconsolidation in the hippocampus. Neuron 36: 527-538. 